\title{
Raman study of stoichiometric and $\mathrm{Zn}$-doped $\mathrm{Fe}_{3} \mathrm{O}_{4}$
}

\author{
Rajeev Gupta, ${ }^{1, *}$ A. K. Sood, ${ }^{1}$ P. Metcalf, ${ }^{2}$ and J. M. Honig ${ }^{2}$ \\ ${ }^{1}$ Department of Physics, Indian Institute of Science, Bangalore, India 560012 \\ ${ }^{2}$ Department of Chemistry, Purdue University, West Lafayette, Indiana 47907
}

\begin{abstract}
We report here our Raman studies on $\mathrm{Fe}_{3-x} \mathrm{Zn}_{x} \mathrm{O}_{4}(x=0,0.015,0.03)$ across the Verwey transition in the temperature range $20-300 \mathrm{~K}$. The changes in Raman spectra as a function of doping show that the changes are gradual for samples with higher Zn doping. Allen's formula has been used to estimate the strength of electronphonon interaction from the observed lineshape parameters. These estimates show that there is strong electronphonon coupling in this system and is highest for the $T_{2 g}^{3}$ mode in comparison to $A_{1 g}$ and $T_{2 g}^{2}$ modes.
\end{abstract}

\section{INTRODUCTION}

Magnetite $\left(\mathrm{Fe}_{3} \mathrm{O}_{4}\right)$ is one of the earliest compounds known to exhibit charge ordering. ${ }^{1}$ Recently it has attracted renewed attention as a result of similar phenomena being observed in other transition metal oxides such as manganites. Stoichiometric $\mathrm{Fe}_{3} \mathrm{O}_{4}$ undergoes a first order metal-insulator (MI) transition (also known as Verwey transition) ${ }^{1}$ as a function of temperature at $121 \mathrm{~K}$. The resistivity increases abruptly by about two orders of magnitude on cooling through the transition temperature $\left(T_{v}\right)$. This transition temperature is extremely sensitive to the oxygen stoichiometry and shifts to lower temperatures as the oxygen deficiency increases, or the system is doped with other transition metals such as zinc or titanium. The nature of the transition also changes from first order to second order beyond a certain doping level $(x>0.015){ }^{2}$

This system has been the subject of many experimental and theoretical studies over the years to understand the Verwey transition. Optical conductivity data ${ }^{3}$ shows transfer of spectral weight from the low energy regions to a peak at 0.6 $\mathrm{eV}$ assigned as a polaronic peak. These data are interpreted in terms of opening up of an optical gap of $140 \mathrm{meV}$. Recent infrared reflectivity experiments of Gasparov et al. ${ }^{4}$ also show gaplike suppression of the optical conductivity below $\sim 125 \mathrm{meV}$. They also observe a peak in conductivty at $\sim 0.6 \mathrm{eV}$ which is linked to twice the polaron binding energy $W_{B}$. Taking the transport to be among $\mathrm{Fe}^{2+}$ and $\mathrm{Fe}^{3+}$, the polaron hopping energy is estimated to be $W_{B} / 2$ $=150 \mathrm{meV}$. This gives the effective mass of carriers to be $\sim 200 m_{e}\left(m_{e}=\right.$ mass of the electron).

Theoretically, the electronic properties have been studied using the local spin density approximation by Zhang et al. ${ }^{5}$ To explain the insulating nature of magnetite, the formation of polarons below $T_{v}$ has been invoked. ${ }^{6,7}$ Polaron formation leads to the renormalization of the electron bandwidth. Band structure calculations have been carried by Ihle and Lorenz ${ }^{8}$ using the Hartree-Fock approximation, invoking only the electronic correlations where the intersite Coulomb interaction is important. Another model to explain the electronic transport by Chakraverty ${ }^{7}$ considers the origin of the Verwey transition to be due to a collective Jahn-Teller distortion. Mott also invoked polarons/bipolarons as the charge carriers in $\mathrm{Fe}_{3} \mathrm{O}_{4}$. Above $T_{v}$, the carriers are localized randomly due to disorder, as in a Wigner glass. As the temperature is increased, some of the polarons dissociate, resulting in hopping type conductivity. Experiments show that the temperature dependence of conductivity is that of an insulator until $400 \mathrm{~K}$, beyond which it becomes metal-like. ${ }^{9}$ Experimental support for the polaron formation exists in the form of shift of the transition temperature as ${ }^{16} \mathrm{O}$ isotope is replaced with ${ }^{18} \mathrm{O}$ isotope. ${ }^{10}$ Shifts in $T_{v}$ as large as $6 \mathrm{~K}$ have been measured for ${ }^{18} \mathrm{O}$ subsitution of $43 \%$. The lattice undergoes a rhombohedral distortion below the transition temperature, ${ }^{11}$ accompanied by a displacement of atoms of the order of 0.01 $\mathrm{nm}$. In the high-temperature phase, several experimental observations such as diffuse scattering of neutrons ${ }^{12}$ around and between the Bragg spots of the cubic phase suggest the formation of polarons and their relation to the Verwey transition. Other physical parameters such as the elastic constant $C_{44}$ (Ref. 13) and magnetic anistropy ${ }^{14}$ show an anomaly near the Verwey transition. This shows the underlying role that the lattice plays across the Verwey transition. Zinc substitution at $A$ sites $^{17}$ affects the transport properties because the divalent $\mathrm{Zn}$ replaces trivalent $\mathrm{Fe}$ on the $A$ sites. Electroneutrality arguments imply a corresponding number of divalent $\mathrm{Fe}$ ions on $B$ sites must be converted to trivalent state, thus diminishing the charge carrier concentration.

There exists several Raman measurements on magnetite, including their temperature dependence to understand the role of the lattice and hence phonons across the Verwey transition. In the early work of Verble ${ }^{18}$ on naturally occuring and synthetic $\mathrm{Fe}_{3} \mathrm{O}_{4}$ crystals, only the $A_{1 g}$ mode at $680 \mathrm{~cm}^{-1}$ was followed as a function of temperature. Although no phonon anomaly was observed in the line shape parameters, the linewidths were large $\left(\sim 30 \mathrm{~cm}^{-1}\right)$ even at low temperatures. This was interpreted in terms of the presence of static electronic disorder due to the random arrangement of $\mathrm{Fe}^{2+}$ and $\mathrm{Fe}^{3+}$ on the $B$ sites and the dynamic disorder due to the hopping of polarons from $\mathrm{Fe}^{2+}$ to $\mathrm{Fe}^{3+}$ sites. Unpolarized Raman measurements on polycrystalline $\mathrm{Fe}_{3} \mathrm{O}_{4}$ by Graves et al. ${ }^{19}$ showed six Raman bands at 706 $\left(A_{1 g}\right), 570\left(A_{1 g}\right), 666\left(E_{g}\right), 336\left(E_{g}\right), 490\left(T_{2 g}\right), \quad$ and $226\left(T_{2 g}\right) \mathrm{cm}^{-1}$. The symmetries were assigned in analogy to the polarized Raman study on $\mathrm{NiFe}_{2} \mathrm{O}_{4}$. Degiorgi et al. ${ }^{20}$ carried out Raman and reflectivity measurements above and below $T_{v}$ on natural single crystals of $\mathrm{Fe}_{3} \mathrm{O}_{4}$. In this work for $T>T_{v}$, four Raman modes were observed at 672, 542, 410, 
and $318 \mathrm{~cm}^{-1}$. Another band observed near $470 \mathrm{~cm}^{-1}$ was assigned to an optical magnon excitation on the basis of neutron scattering experiments, ${ }^{21}$ which showed a zone center optical magnon at $465 \pm 8 \mathrm{~cm}^{-1}$. The magnon mode was also seen in the Raman experiments of Hart et al. ${ }^{22}$ Apart from the vibrational and magnon modes, they observed fluorescence when $514.5 \mathrm{~nm}$ or $488 \mathrm{~nm}$ wavelengths were used to excite the Raman modes. The maximum of the fluorescence band occurred at $\sim 2.28 \mathrm{eV}$ at $300 \mathrm{~K}$ and shifted to lower energy as the temperature was lowered. Its origin was traced to electronic transitions across the spin-split gap at the $B$ (octahedral) sites. Very recently, Gasparov et al. ${ }^{4}$ (we became aware of this work only after the completion of our experiments) also followed the $A_{1 g}$ mode at $680 \mathrm{~cm}^{-1}$ as a function of temperature and found drastic changes in the linewidth across $T_{v}$. It was suggested that the sudden drop in the Raman mode frequency and the increase in the linewidth at the Verwey transition could be due to the coupling of the $A_{1 \mathrm{~g}}$ phonon to the structural displacements occuring at $T_{v} \cdot{ }^{4}$ In the present comprehensive Raman study on magnetite, we examined all the Raman modes as a function of composition and temperature across the Verwey transition. One motivation to study $\mathrm{Fe}_{3} \mathrm{O}_{4}$ in a detailed manner was that carefully characterized crystals of high quality were now available. The single crystals used in our studies were obtained by proper annealing procedures and hence the stoichiometry quoted is same throughout the crystal. We have carried out measurements on very high quality single crystals of $\mathrm{Fe}_{3-x} \mathrm{Zn}_{x} \mathrm{O}_{4} \quad(x=0,0.015$ and 0.030$)$ and $\mathrm{Fe}_{3(1-\delta)} \mathrm{O}_{4}(\delta$ $\left.=0.01, T_{v}=87 \mathrm{~K}\right)$. The objective in carrying out the present study is to address the role of phonons in the Verwey transition, with emphasis on studying the changes in peak positions and linewidths of the Raman active modes. From the measured linewidths and peak positions of Raman modes, we have estimated the magnitude of the electron-phonon coupling parameter $\lambda$ for different symmetry phonons. It will be shown that the $T_{2 g}^{3}$ mode has the highest $\lambda$.

\section{EXPERIMENTAL DETAILS}

The well characterized single crystals of magnetite used for the experiments were grown by the standard skull melt technique. Detailed characterization studies have been reported earlier. ${ }^{23}$ For the sample with $x=0$ and a transition temperature $\sim 121 \mathrm{~K}$, there is a discontinous jump in the resistivity at $T_{v}$. The jump in resistivity for sample $2(x$ $=0.015)$ with $T_{v}=106 \mathrm{~K}$ is much smaller than for sample 1. Sample $3(x=0.030)$ with $T_{v}=87 \mathrm{~K}$ also stays insulating at all temperatures but without any discontinuity in resistivity at $T_{v}$. A (100) crystalline face of a single crystal of size $2 \times 2 \times 2 \mathrm{~mm}^{3}$ was polished using diamond paste (particle size $\sim 1 \mu \mathrm{m})$ and mounted on the cold finger of the closed cycle helium cryostat (RMC model 22C CRYODYNE) using thermally cycled GE (M/s. General Electric, USA) varnish. Raman spectra were recorded in the temperature range 27 to $300 \mathrm{~K}$. The spectra were recorded in the spectral range of 150 to $800 \mathrm{~cm}^{-1}$ in the back scattering geometry using a DILOR $X Y$ spectrometer equipped with a liquid nitrogen cooled charge coupled device detector. To remove any ambi- guity in the measurement of peak positions a standard neon line spectrum was recorded in each spectral range, and the magnetite spectra were corrected for shifts, if any. Although the incident light was polarized, no analyzer was introduced in the scattered light, as the Raman signals were weak. The Rayleigh background was minimized by using a thin copper wedge between the cold finger and the sample to keep the specular reflection out of the spectrometer. An argon ion laser line at $514.5 \mathrm{~nm}$ was used as the excitation wavelength, with a laser power of $\sim 25 \mathrm{~mW}$ focused to a spot of $\sim 40$ microns on the sample. The quoted temperatures are those measured on the cold finger using a Platinum-100 sensor coupled to a home made temperature controller. The sample temperature is higher by about $20 \mathrm{~K}$ primarily due to laser heating as compared to the quoted temperatures. The temperatures are accurate to $\sim 2 \mathrm{~K}$.

\section{RAMAN MODES}

In the temperature regime $T>T_{v}$, the primitive unit cell is cubic with two formula units per unit cell. The space group is $O_{h}^{7}(F d 3 m)$ and the irreducible representation at the zone center is given by ${ }^{24}$

$$
\begin{aligned}
\Gamma= & A_{1 g}(R)+E_{g}(R)+T_{1 g}+3 T_{2 g}(R)+2 A_{2 u}+2 E_{u} \\
& +5 T_{1 u}(I R)+2 T_{2 u}
\end{aligned}
$$

where R and IR denote Raman and infrared activity of the modes. The $T_{1 g}$ mode is silent; thus there are five Raman active modes at room temperature: $A_{1 g}(\omega$ $\left.=669 \mathrm{~cm}^{-1}\right), E_{g}\left(\omega=410 \mathrm{~cm}^{-1}\right), \quad$ and $3 T_{2 g}\left[\omega\left(T_{2 g}^{1}\right)\right.$ $\left.=193 \mathrm{~cm}^{-1}, \omega\left(T_{2 g}^{2}\right)=540 \mathrm{~cm}^{-1}, \omega\left(T_{2 g}^{3}\right)=300 \mathrm{~cm}^{-1}\right]$.

Verble had assigned the Raman mode observed at $300 \mathrm{~cm}^{-1}$ to be $T_{2 g}^{1}$. However, Gasparov et al. ${ }^{4}$ assign the mode at $193 \mathrm{~cm}^{-1}$ observed at room temperature to be $T_{2 g}^{1}$ and attributed the $300 \mathrm{~cm}^{-1}$ mode as arising from the lowering of the symmetry below $T_{v}$. Although $\mathrm{Fe}_{3} \mathrm{O}_{4}$ is not a molecular solid, the treatment of vibrational modes of ferrites in terms of a molecular model, as proposed by Waldron, ${ }^{25}$ makes it easy to visualise the eigenmodes of different Raman active modes.

As the temperature is lowered below $T_{v}$, the symmetry of the crystal is lowered. Verble ${ }^{18}$ treated the low temperature phase as orthorhombic with a body centered space group $D_{2 h}^{28}($ Imma $)$ as proposed by Hamilton ${ }^{26}$ on the basis of neutron diffraction studies. The unit cell doubles along the $c$ direction and the diagonals of the earlier cubic unit cell become the faces of the low temperature unit cell. In this phase [space group $D_{2 h}^{28}($ Imma $)$ ] there are fifteen Raman active modes. Recent neutron scattering measurements ${ }^{12,27}$ show, however, that the crystal structure below $T_{v}$ is monoclinic, with space group $(C c)$. The analysis of the low-temperature structure of magnetite is complicated by the presence of a high degree of pseudosymmetry and a complete refinement has not been possible from the neutron diffraction data. However, the presence of a rhombohedral distortion of the cubic unit cell, doubling of the cell along the $c$ axis and the existence of $c$-glide point to a monoclinic structure have 

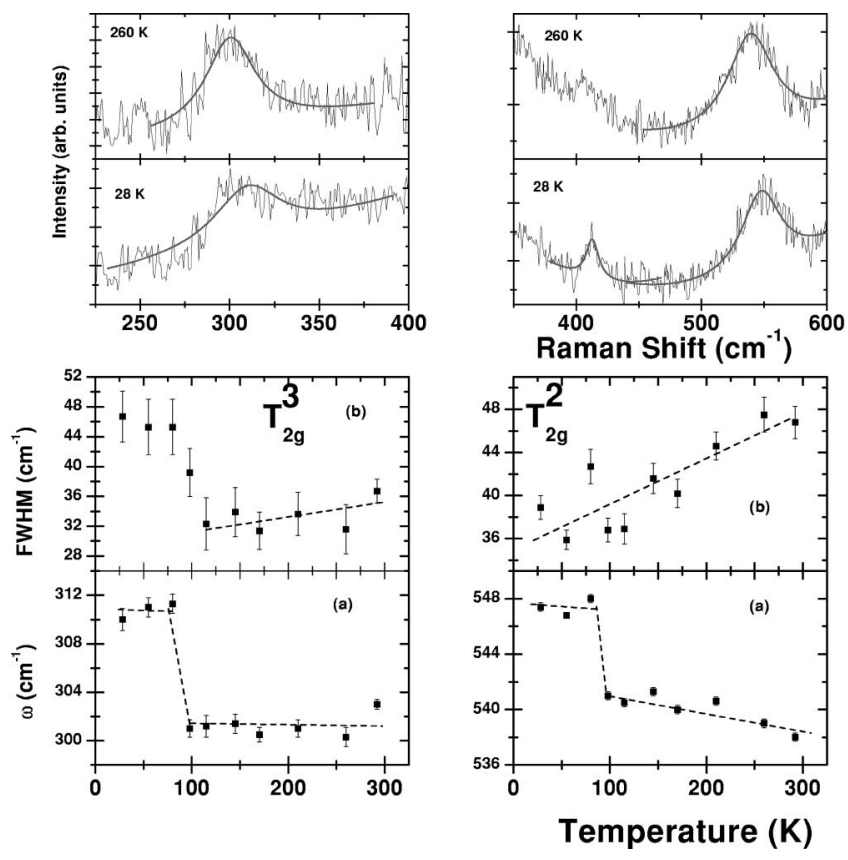

been demonstrated. Of the two possible space groups suggested for the low-temperature phase by neutron scattering, $D_{2 h}^{11}(P b c m)$ and $C_{2 v}^{2}\left(P m c 2_{1}\right)$, the latter was ruled out by Gasparov et al. ${ }^{4}$ on the basis of Raman and infrared experiments. The space group $C_{2 v}^{2}$ does not have an inversion center, so that the modes should be both Raman and infrared active, which was not the case experimentally. For the space group Pmca, the unit cell contains 56 atoms and there are 78 Raman active and 72 infrared active modes. Here it should be noted that though the number of modes below $T_{v}$ are different in the pictures of Verble ${ }^{18}$ and Gasparov et al. ${ }^{4}$ the symmetry of the modes is the same in the two possible lowtemperature space groups. However, it is clear that the lowtemperature phase with lower symmetry has a large number of modes. Some of these arise due to the folding of the Brillioun zone consequent to the doubling of the unit cell upon charge ordering. The degeneracy of the $T_{2 g}$ and $E_{g}$ modes gets lifted below $T_{v}$, i.e., the $T_{2 g}$ modes split into $B_{1 g}+B_{2 g}+B_{3 g}$. The $A_{1 g}$ mode in the cubic phase persists below $T_{v}$ as an $A_{g}$ mode.

\section{RESULTS}

We will now present Raman spectra for the four samples described earlier. The top panels in Fig. 1 shows the Raman spectra of stoichiometric $\mathrm{Fe}_{3} \mathrm{O}_{4}$ in the spectral range 150-800 $\mathrm{cm}^{-1}$ at two temperatures. In this region we observe four modes at room temperature: the strongest $A_{1 g}$ mode at $669 \mathrm{~cm}^{-1}, T_{2 g}$ modes at 300 and $540 \mathrm{~cm}^{-1}$, and a weak $E_{g}$ mode at $410 \mathrm{~cm}^{-1}$. We did not observe the weak $T_{2 g}^{1}$ mode reported in earlier studies at $193 \mathrm{~cm}^{-1}$ and the mode at $\sim 470 \mathrm{~cm}^{-1}$ attributed to magnons. The symmetry assignment has been done as per the earlier assignments by Verble $^{18}$ and Gasparov et al. ${ }^{4}$ (The modes will be either referred by there symmetry assignments or their room temperature peak positions.) Due to the weak Raman signal, we

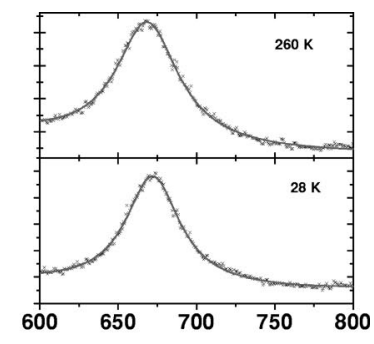

FIG. 1. Raman spectra for sample 1, $\quad \mathrm{Fe}_{3-x} \mathrm{Zn}_{x} \mathrm{O}_{4}(x=0)$ with $T_{v}=121 \mathrm{~K}$ at selected temperatures in the range 200-800 $\mathrm{cm}^{-1}$. The thick smooth line shows the Lorentzian fits to the data. The lower panels show the variation of the lineshape parameters of the $A_{1 g}$ and $T_{2 g}$ modes as a function of temperature. The dashed lines are drawn as a guide to the eyes.

could not clearly identify additional Raman modes expected at $T<T_{v}$. The observed data were fitted to a sum of appropriate number of Lorentzians and a second degree polynomial baseline. The lineshape parameters, peak position $(\omega)$ and full width at half maximum $\Gamma$ (FWHM) thus obtained as a function of temperature are presented in bottom panels of Fig. 1. Here the dashed lines are drawn as a guide to the eyes. The intensity did not show any specific temperature dependence, possibly due to experimental difficulties (i.e., we do not have an internal reference to normalize the intensities). We note that the temperature dependence of the $A_{1 g}$ mode frequency is similar to the one observed by Gasparov et $a l .{ }^{4}$ The line widths of the $A_{1 g}$ mode in Fig. 1 is comparable to that of Gasparov et al. ${ }^{4}$ at room temperature. However, this linewidth in our case is much larger than that observed by Gasparov et al. ${ }^{4}$ at low temperature. The larger linewidth at low temperature could possibly arise also due to the polishing-induced strain in the surface layer.

Figure 2 (top panels) shows the Raman spectra for $\mathrm{Fe}_{3-x} \mathrm{Zn}_{x} \mathrm{O}_{4}\left(x=0.015, T_{v}=106 \mathrm{~K}\right)$ at two temperatures in the spectral range $150-800 \mathrm{~cm}^{-1}$. In this sample we observe three modes at $307\left(T_{2 g}^{3}\right), 538\left(T_{2 g}^{2}\right)$, and $669 \mathrm{~cm}^{-1}\left(A_{1 g}\right)$. The thick smooth lines are fits to the Lorentzian functions. The $E_{g}$ mode at $410 \mathrm{~cm}^{-1}$ is not observed in the spectra. The temperature dependence of $\omega$ and FWHM are plotted for $A_{1 g}, T_{2 g}^{2}$, and $T_{2 g}^{3}$ modes in Fig. 2 (bottom panels). Here also the dashed lines are a guide to the eyes.

Figure 3 shows the Raman spectra of sample 3, $\mathrm{Fe}_{3-x} \mathrm{Zn}_{x} \mathrm{O}_{4}\left(x=0.029, T_{v}=87 \mathrm{~K}\right)$ at a few temperatures in the spectral range $500-800 \mathrm{~cm}^{-1}$. In this sample the intensity of the modes is weaker in comparison with samples 1 and 2 , so much so that only two modes were observed at $671 \mathrm{~cm}^{-1}\left(A_{1 g}\right)$ and $540\left(T_{2 g}^{2}\right)$. The $E_{g}$ and $T_{2 g}^{3}$ modes were not seen. The temperature variation of $\omega$ and the FWHM are plotted in Fig. 3. 

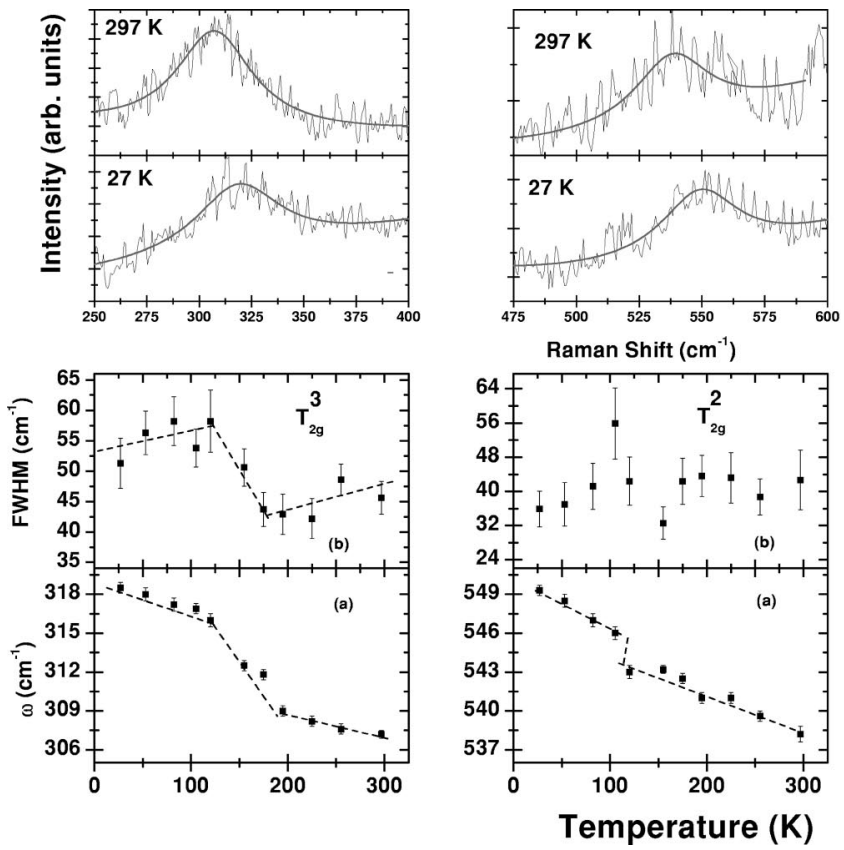

Apart from the stoichiometric and zinc doped $\mathrm{Fe}_{3} \mathrm{O}_{4}$, we have also carried out experiments on nonstoichiometric $\mathrm{Fe}_{3} \mathrm{O}_{4}$ with changes of $\delta$ in $\mathrm{Fe}_{3(1-\delta)} \mathrm{O}_{4}$. For $\delta=0.01$, sample 4 shows a second order Verwey transition at $87 \mathrm{~K}$. The Raman spectra of sample 4 are shown in Fig. 4 at a few selected temperatures. The modes occur at 232, 253, 299, 312, 424, 641 , and $685 \mathrm{~cm}^{-1}$. It appears that most of these modes are closer to those of $\mathrm{Fe}_{2} \mathrm{O}_{3}$ which has seven Raman modes at ${ }^{28}$ 226, 245, 293, 298, 413, 500, and $612 \mathrm{~cm}^{-1}$. This is not surprising because for $\delta>\delta_{c} \sim 0.0039$, the samples cannot be readily produced as single phases because of the proximity of the $\mathrm{Fe}_{3} \mathrm{O}_{4}-\mathrm{Fe}_{2} \mathrm{O}_{3}$ phase boundary. ${ }^{17}$ In contrast to the stoichiometric and $\mathrm{Zn}$ doped $\mathrm{Fe}_{3} \mathrm{O}_{4}$, the $A_{1 g}$ mode at $\sim 685 \mathrm{~cm}^{-1}$ is very weak. More work is needed to understand the Raman spectra of non-stoichiometric $\mathrm{Fe}_{3} \mathrm{O}_{4}$. We
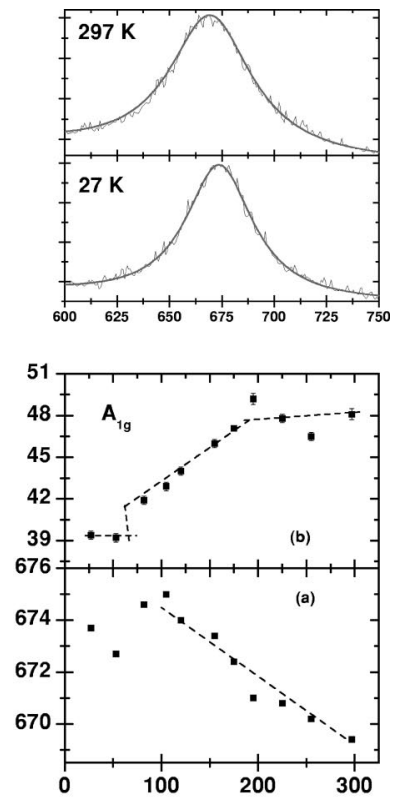

will, therefore, discuss our results only for stoichiometric and $\mathrm{Zn}$ doped samples.

\section{DISCUSSION}

For a systematic behavior of the peak positions of the observed modes as a function of $\mathrm{Zn}$ doping in $\mathrm{Fe}_{3-x} \mathrm{Zn}_{x} \mathrm{O}_{4}$, we have plotted $\omega(x)-\omega(x=0) / \omega(x=o)$ versus $x$ for the $A_{1 g}, T_{2 g}^{2}$, and $T_{2 g}^{3}$ modes at 30 and $300 \mathrm{~K}$ in Figs. 5(a) and 5 (b), respectively. The changes in $\omega$ with $T$ also depends on the doping as shown in Fig. 5(c), where the quantity $\Delta \omega / \omega(300 \mathrm{~K}) \quad[\Delta \omega=\omega(30 \mathrm{~K})-\omega(300 \mathrm{~K})]$ has been plotted versus $x$ for all the $A_{1 g}, T_{2 g}^{2}$, and $T_{2 g}^{3}$ modes.

In stoichiometric $\mathrm{Fe}_{3} \mathrm{O}_{4}$, the $A_{1 g}$ mode frequency $\omega$ shows an abrupt change by $\sim 3 \mathrm{~cm}^{-1}$ near $T_{v}$ [see Fig.
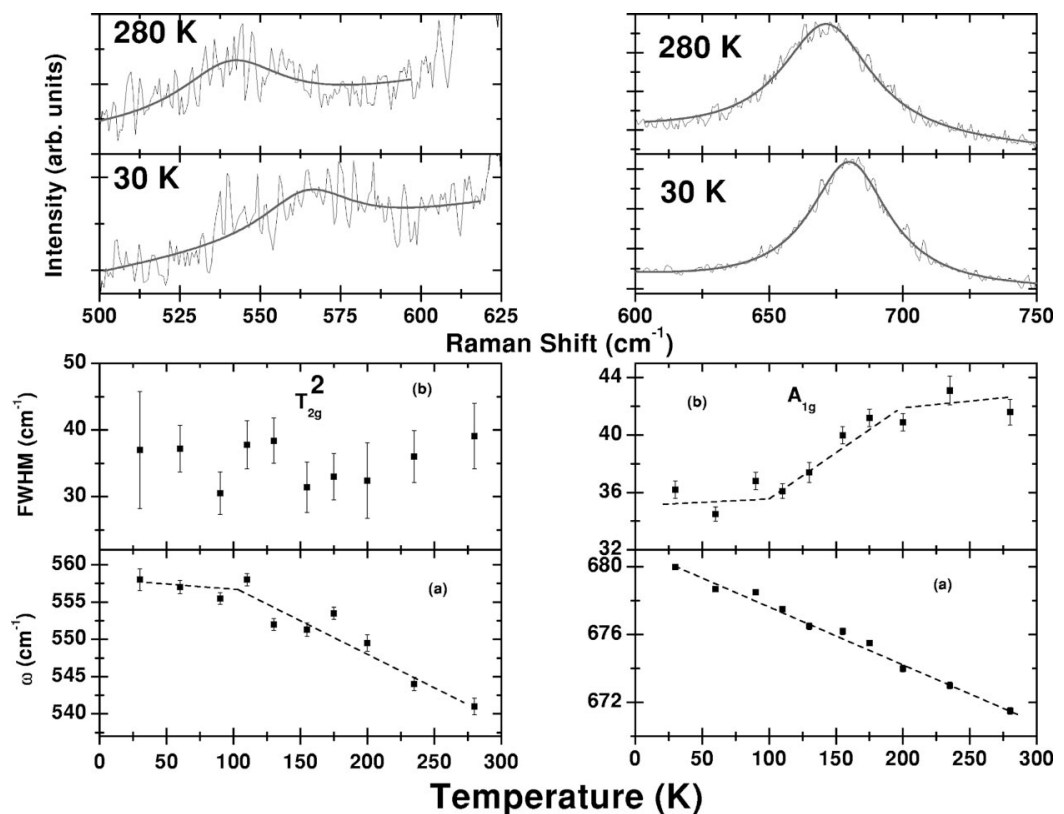

FIG. 3. Raman spectra in the range 400-800 $\mathrm{cm}^{-1}$ at selected temperatures for the sample $3, \mathrm{Fe}_{3-x} \mathrm{Zn}_{x} \mathrm{O}_{4}(x=0.03)$ with $T_{v}=87 \mathrm{~K}$. The lower panels show the temperature dependence of the peak position and linewidth for the $T_{2 g}^{2}$ and $A_{1 g}$ modes. 


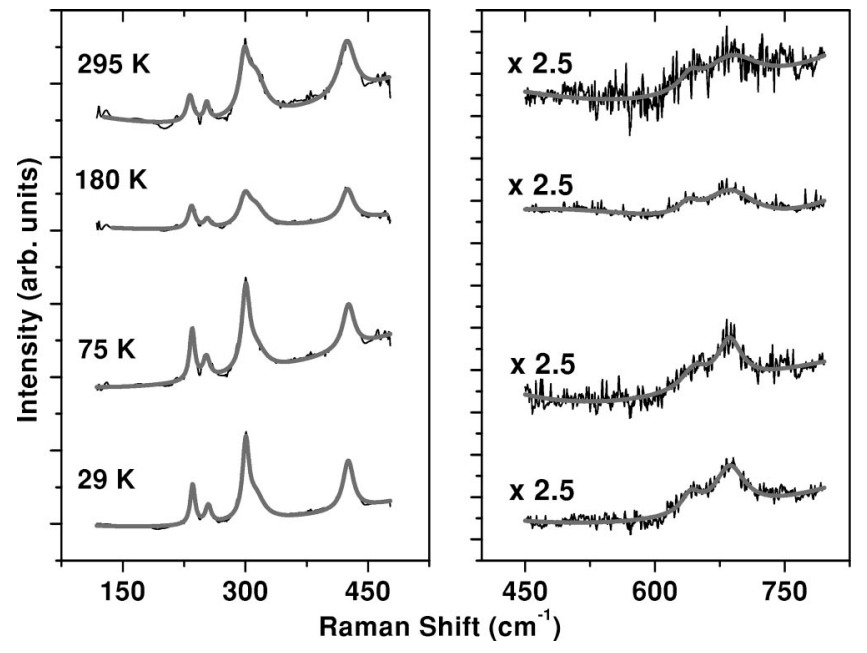

FIG. 4. Raman spectra in the range $100-800 \mathrm{~cm}^{-1}$ at selected temperatures for the sample $\mathrm{Fe}_{3(1-\delta)} \mathrm{O}_{4}(\delta=0.01)$ with $T_{v}=87 \mathrm{~K}$.

1(a)]. Above $T_{v}, \omega$ is nearly flat as the temperature is raised up to $300 \mathrm{~K}$. As compared to $A_{1 g}$ mode, the $T_{2 g}^{2}$ mode at $540 \mathrm{~cm}^{-1}$ and the $T_{2 g}^{3}$ mode at $300 \mathrm{~cm}^{-1}$ soften to a much greater extent $\left(\sim 10 \mathrm{~cm}^{-1}\right)$, near $T_{v}$ (Fig. 1). The changes are more gradual in Zn doped samples, as seen in Figs. 2 and 3 , and the magnitude of the changes between 30 and $300 \mathrm{~K}$ is higher in $\mathrm{Zn}$ doped samples as shown in Fig. 5(c). At a given temperature, the mode frequency increases with $x$ for all the three modes, the change being largest for the $T_{2 g}^{3}$ mode as quantified in Figs. 5(a) and 5(b). Magnetite crystallizes in the inverse spinel cubic structure with 8 formula units per unit cell and can be thought of as made up of $\mathrm{Fe}^{3+}$ and $\mathrm{Fe}^{2+}$ ions in the ratio of 2:1 and arranged on two interpenetrating lattices $A$ and $B$. The $A$ sites are tetrahedrally coordinated with respect to oxygen while the $B$ sites are octahedrally coordinated. ${ }^{1,15,16}$ For the $A$ sites, the interstices of coordination oxygen tetrahedra are too small to accomodate larger $\mathrm{Fe}^{2+}$ ions and hence these sites are occupied only by $\mathrm{Fe}^{3+}$ species, whereas the $B$ sites are occupied by $\mathrm{Fe}^{2+}$ and $\mathrm{Fe}^{3+}$. Since $\mathrm{Zn}$ replaces $\mathrm{Fe}^{3+}$ at the $A$ sites ${ }^{17}$ around which the vibrating oxygen ions are situated, the changes in $\omega$ can be expected. The increase in $\omega$ would imply that the Fe-O bond length should decrease with $x$. This is perhaps not so because the lattice constant of $\mathrm{Fe}_{3} \mathrm{O}_{4}$ increases with $\mathrm{Zn}$ doping. ${ }^{27}$ This would suggest that the increase in $\omega$ with $x$ should derive from the enhancement of the $\mathrm{Fe}-\mathrm{O}$ bond strength due to increased effective charge. The reason for $\Delta \omega / \omega(300 \mathrm{~K})$ $[\Delta \omega=\omega(30 \mathrm{~K})-\omega(300 \mathrm{~K})]$ being higher for $T_{2 g}^{3}$ mode, followed by $T_{2 g}^{2}$ and $A_{1 g}$ is that the electron-phonon interaction is largest for $T_{2 g}^{3}$, as will be discussed later.

The temperature dependence of phonon frequency can arise due to the following reasons. (a) As the lattice expands due to thermal expansion, resulting in the lowering of the force constants between ions and hence lowering of the mode frequency. Quantitatively, this effect can be expressed in terms of the mode Grüneisen parameter as

$$
\omega_{i}(T)-\omega_{i}(0)=-\gamma_{i}\left(\frac{\Delta V}{V}\right),
$$

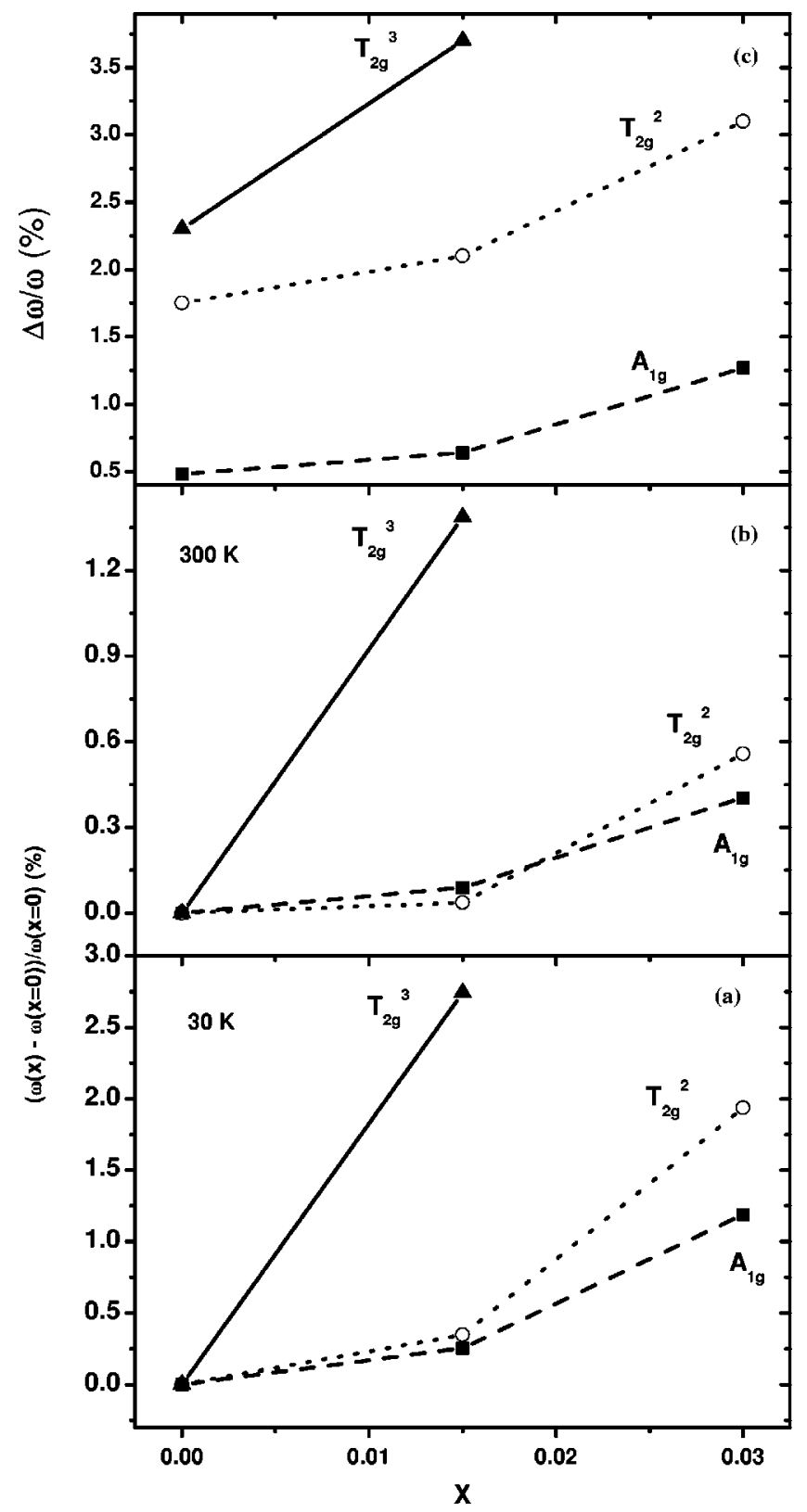

FIG. 5. Plots of, $[\omega(x)-\omega(x=0)] / \omega(x=0)$ versus $x$ (doping) for $A_{1 g}, T_{2 g}^{2}$, and $T_{2 g}^{3}$ modes at 30 and $300 \mathrm{~K}$. Also shown, $[\omega(30 \mathrm{~K})-\omega(300 \mathrm{~K})] / \omega(300 \mathrm{~K})$ versus $x$ for the $A_{1 g}, T_{2 g}^{2}$, and $T_{2 g}^{3}$ modes.

where $\gamma_{i}$ is the Grüneisen parameter for the $i$ th phonon mode and $\Delta V / V$ is the fractional volume change. Typically for perovskites such as $\mathrm{SrTiO}_{3}, \gamma_{i} \sim 1.6 .{ }^{29}$ The temperature dependence of the lattice parameter measured using neutron scattering experiments ${ }^{27}$ show that the cube length expands by $0.1 \%$ in the temperature range of 125 to $275 \mathrm{~K}$, with a jump of $0.05 \%$ at $T_{v}$. This does not account for the magnitude and trend of the observed changes in $\omega$ (For $T<T_{v}$, there is a lattice expansion which can cause softening instead of hardening as seen in our experiments).

(b) A second contribution to $\Delta \omega$ arises from the intrinsic anharmonic interactions at constant volume due to the change in the phonon population. In the temperature regime 
$T<\Theta_{D}$, the Debye temperature, cubic anharmonicity giving rise to the decay of a phonon into two phonons dominates the self-energy of the quasiparticle (phonon). The real part of self-energy results in the shift of the frequency and the imaginary part leads to broadening of the linewidths. Phenomenologically, assuming the decay of a phonon of frequency $\omega$ into two phonons of frequencies $\omega_{1}$ and $\omega_{2}(=\omega$ $\left.-\omega_{1}\right),{ }^{30}$ we write

$$
\Delta \omega_{\mathrm{anh}}=A\left[n\left(\omega_{1}\right)+n\left(\omega_{2}\right)+1\right],
$$

where $A$ is a constant representing the phonon-phonon interaction. $\mathrm{Fe}_{3} \mathrm{O}_{4}$ has a Debye temperature of $\Theta_{D} \sim 450 \mathrm{~K} .{ }^{27} \mathrm{We}$ believe that the anharmonic contributions are very small because the $A_{1 g}$ mode frequency for sample 1 changes by less than $1 \mathrm{~cm}^{-\mathrm{f}}$ in the temperature range of 125 to $300 \mathrm{~K}$ (Fig. 1). This is also the case for all the modes in nonstoichiometric sample 4.

(c) Another contribution to changes in mode frequency can arise from the electron-phonon interaction, e.g., changes in the density of states at the Fermi level $N\left(E_{F}\right)$ can affect the phonon softening in a subtle manner which we discuss later, after presenting our observations about the FWHM of the modes observed.

A common feature to all the Raman modes in the three samples $(1-3)$ is that the linewidths are very large $\left(\sim 30-40 \mathrm{~cm}^{-1}\right)$ even at low temperature, as was also noted in the earlier reports. ${ }^{4,18}$ Verble interpreted the large linewidth of the $A_{1 g}$ at low temperatures as due to electronic disorder arising as a result of random arrangement of $\mathrm{Fe}^{2+}$ and $\mathrm{Fe}^{3+}$ ions on the $B$ sites. ${ }^{18}$

Another striking feature in Figs. 1 and 2 is that the linewidth of $T_{2 g}^{3}$ mode is higher at low temperature as compared to its room temperature value. At first sight this appears anomalous. The Infrared active mode in $\mathrm{Fe}_{3} \mathrm{O}_{4}$ at $350 \mathrm{~cm}^{-1}$ also shows such an anomaly; this was interpreted in terms of lowering of the crystal symmetry below $T_{v}$ which lifts the degeneracy of the triply degenerate mode. ${ }^{4}$ The neutron scattering experiments show that the $\mathrm{Fe}-\mathrm{O}$ distances for $A$ and $B$ sites are within the estimated standard deviations; therefore the splittings are small, ${ }^{11}$ giving rise to a large apparent width. We believe that a similar explaination holds for the threefold degenerate Raman $T_{2 g}^{3}$ mode, where the lowering of crystal symmetry splits the $T_{2 g}^{3}$ mode into nondegenerate $B_{1 g}+B_{2 g}+B_{3 g}$ modes. In view of this, we will only discuss the linewidth of the $A_{1 g}$ mode at all temperatures and $T_{2 g}^{2}$ and $T_{2 g}^{3}$ modes at room temperature in a more quantitative manner.

As mentioned earlier, the anharmonic contribution is not adequate to explain the temperature dependence of $\omega$ and $\Gamma$. We believe that the major contribution to the linewidth arises from strong electron-phonon interaction in $\mathrm{Fe}_{3} \mathrm{O}_{4}$ associated with the decay of phonon into electron-hole pair. Allen ${ }^{31}$ had shown that for metallic systems, the strength $\lambda$ of the electron-phonon interaction can be estimated from the average of the phonon linewidth over all $\vec{q}$ wave vectors. For ordered systems, energy and momentum conservation allows excitation of only $|\vec{q}| \sim 0$ phonons which cannot decay in an

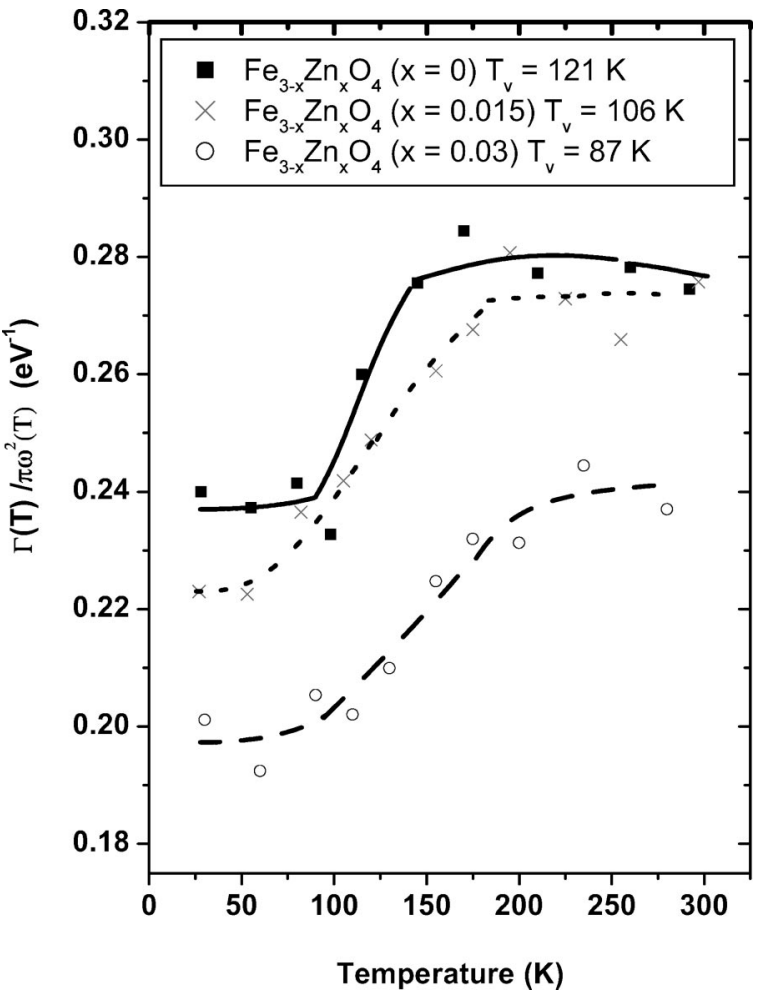

FIG. $6 . \Gamma / \pi \omega^{2}$ for the stoichiometric and zinc doped samples as a function of temperature. The lines are drawn as a guide to the eyes.

intraband electron-hole pair excitation. Therefore, Allen's prescription to deduce $\lambda$ from Raman linewidths will not be applicable. However, in the presence of disorder, wavevector conservation rule is relaxed and one can apply Allen's formula to the Raman linewidths. Such is the case in $\mathrm{Fe}_{3} \mathrm{O}_{4}$ above the order-disorder transition temperature $T_{v} \cdot{ }^{18}$ For a $m$ th phonon, the FWHM $\Gamma_{m}$ and frequency $\omega_{m}$ are related by Allen's formula

$$
\frac{\Gamma_{m}}{\omega_{m}^{2}}=\frac{2 \pi}{g_{m}} \lambda_{m} N\left(E_{F}\right)
$$

where $\lambda_{m}$ is the electron-phonon coupling strength, $g_{m}$ is the degeneracy of the $m$ th mode, and $N\left(E_{F}\right)$ is the density of states at the Fermi level.

Figure 6 shows the temperature dependence of $\Gamma(T) / \omega^{2}$ for the $A_{1 g}$ mode in $\mathrm{Fe}_{3} \mathrm{O}_{4}, \mathrm{Fe}_{3-x} \mathrm{Zn}_{x} \mathrm{O}_{4}(x$ $=0.015), \mathrm{Fe}_{3-x} \mathrm{Zn}_{x} \mathrm{O}_{4}(x=0.03)$. Since the observed linewidths are much larger than the instrumental resolution, we have not corrected the linewidths to deconvolute the instrumental resolution width. As noted before, there can be some contribution to the linewidth from the strain induced by polishing the crystal surface. Since this contribution is similar for all the crystals studied and is temperature independent, it is justifiable to compare the temperayure dependence of $\Gamma(T) / \omega^{2}$ for three samples, as done in Fig. 6. It is seen that the changes are more gradual for zinc doped $\mathrm{Fe}_{3} \mathrm{O}_{4}$ in comparison to the stoichiometric compound. This is in line with the fact that the transition becomes second order on zinc 
TABLE I. $\Gamma / \omega^{2}$ (at $300 \mathrm{~K}$ ), $\lambda N\left(E_{F}\right)$, and deformation potential $D$ for the $A_{1 g}, T_{2 g}^{2}$, and $T_{2 g}^{3}$ modes for samples 1,2 and 3 .

\begin{tabular}{lccc}
\hline \hline Mode & $\frac{\Gamma}{\omega^{2}}(\mathrm{eV})^{-1}$ & $\lambda$ & $D(\mathrm{eV} / \AA)$ \\
\hline$A_{1 g}$ & 0.86 & 0.045 & 0.11 \\
$T_{2 g}^{2}$ & 1.30 & 0.20 & 0.18 \\
$T_{2 g}^{3}$ & 3.22 & 0.51 & 0.16 \\
Mode & $\Gamma$ & & \\
& $\frac{\Gamma}{\omega^{2}}(\mathrm{eV})^{-1}$ & $\lambda$ & $D(\mathrm{eV} / \AA)$ \\
$A_{1 g}$ & 0.87 & 0.045 & 0.11 \\
$T_{2 g}^{2}$ & 1.19 & 0.19 & 0.18 \\
$T_{2 g}^{3}$ & 3.90 & 0.62 & 0.18 \\
Mode & $\Gamma$ & & \\
& $\frac{\Gamma}{\omega^{2}}(\mathrm{eV})^{-1}$ & $\lambda$ & 0.10 \\
$A_{1 g}$ & 0.74 & 0.04 & 0.17 \\
$T_{2 g}^{2}$ & 1.08 & 0.17 & \\
$T_{2 g}^{3}$ & & & 0.10 \\
\hline \hline
\end{tabular}

doping higher than a critical concentration. The decrease in $\Gamma(T) / \omega^{2}$ near $T_{v}$ clearly shows $N\left(E_{F}\right)$ [see Eq. (4)] decreases by $\sim 15$ to $20 \%$. Such decrease in the density of states is also seen in photoemission experiments. ${ }^{32,33}$

Taking the calculated value ${ }^{5}$ of $N\left(E_{F}\right)=3$ states/eV per $\mathrm{Fe}$, at room temperature for all samples, and using $\Gamma / \omega^{2}$ values at room temperature, we have estimated the electronphonon coupling constant for the three modes, as shown in Table I. The value of $\lambda$ seems reasonable [compared to the ones in doped $\mathrm{C}_{60}$ (Ref. 34)]. The most interesting aspect is that $\lambda$ is highest for $T_{2 g}^{3}$ mode $\lambda\left[T_{2 g}^{3}\right] / \lambda\left[T_{2 g}^{2}\right]$ $=2.6, \lambda\left[T_{2 g}^{3}\right] / \lambda\left[A_{1 g}\right]=11.4$. This can be understood by re- alizing that the electronic states have $T_{2 g}$ symmetry near the Fermi level ${ }^{5}$ and hence would couple strongly to $T_{2 g}$ phonons as compared to $A_{1 g}$ phonons.

We have also attempted to estimate the deformation potential $D$ of the three modes from the data. It has been shown that the electron-phonon coupling constant is related to $D$ by $^{34}$

$$
\lambda_{i}=\frac{5}{6} \frac{N\left(E_{F}\right)}{M \omega_{i}^{2}} D_{i}^{2} .
$$

The estimated values of $D$ are also given in Table I, showing that $D \sim 0.18 \mathrm{eV} / \AA$ for the $T_{2 g}$ modes and $\sim 0.11 \mathrm{eV} / \AA$ for the $A_{1 g}$ mode.

\section{CONCLUSIONS}

Our Raman measurements on stoichiometric and doped $\mathrm{Fe}_{3} \mathrm{O}_{4}$ show that strong electron-phonon coupling occurs in these systems. This scenario is in agreement with the polaronic picture of transport in these systems. The changes observed in phonon line shape parameters are gradual for higher $\mathrm{Zn}$ doped samples as expected for systems showing a crossover from a first order transition to a higher order transition. We do not have a satisfactory explanation to understand the origin of large linewidth of $A_{1 g}$ mode even at low temperatures. More experiments are required to understand the nature of disorder. A new noteworthy finding in our present study is that the $T_{2 g}^{3}$ mode has the highest electronphonon coupling as compared to the $A_{1 g}$ and $T_{2 g}^{2}$ modes. It will be very interesting to study the dispersion of the $T_{2 g}^{3}$ phonons as a function of temperature using inelastic neutron scattering.

\section{ACKNOWLEDGMENTS}

A.K.S. thanks Department of Science and Technology, India for financial assistance.
*Present address: Dept. of Physics, Pennsylvania State University, University Park, PA 16802. Email address: rug4@psu.edu

${ }^{1}$ E.J.W. Verwey, Nature (London) 144, 327 (1941); E.J.W. Verwey and P.W. Haaymann, Physica (Amsterdam) 8, 979 (1941).

${ }^{2}$ J.P. Shepherd et al., Phys. Rev. B 43, 8461 (1991).

${ }^{3}$ S.K. Park, T. Ishikawa, and Y. Tokura, Phys. Rev. B 58, 3717 (1998).

${ }^{4}$ L.V. Gasparov et al., Phys. Rev. B 62, 7939 (2000).

${ }^{5}$ Z. Zhang and S. Satpathy, Phys. Rev. B 44, 13319 (1991).

${ }^{6}$ N.F. Mott, in Metal-Insulator Transitions, 2nd ed. (Taylor and Francis, London, 1990), p. 217.

${ }^{7}$ B.K. Chakraverty, Solid State Commun. 15, 1271 (1974).

${ }^{8}$ D. Ihle and B. Lorenz, Philos. Mag. B 42, 337 (1980).

${ }^{9}$ P.A. Miles, W.B. Westphal, and A. von Hippel, Rev. Mod. Phys. 29, 279 (1957).

${ }^{10}$ E.I. Terukov, W. Reichelt, D. Ihle, and H. Oppermann, Phys. Status Solidi B 95, 491 (1979).

${ }^{11}$ M. Iizumi et al., Acta Crystallogr., Sect. B: Struct. Crystallogr. Cryst. Chem. 38, 2121 (1982).

${ }^{12}$ S.M. Shapiro, M. Iizumi, and G. Shirane, Phys. Rev. B 14, 200 (1976).
${ }^{13} \mathrm{~S}$. Chikazumi, in Magnetism and Magnetic Materials, edited by J. J. Becker et al., AIP Conf. Proc. No. 29 (AIP, New York, 1976), p. 382.

${ }^{14}$ K. Abe, Y. Miyamoto, and S. Chikazumi, J. Phys. Soc. Jpn. 41, 1894 (1976).

${ }^{15}$ Z. Kakol, J. Solid State Chem. 88, 104 (1990).

${ }^{16}$ C.N.R. Rao and J. Gopalakrishnan, New Directions in Solid State Chemistry (Cambridge University Press, Cambridge, 1986).

${ }^{17}$ P. Wang, Z. Kakol, M. Wittenauer, and J.M. Honig, Phys. Rev. B 42, 4553 (1990).

${ }^{18}$ J.L. Verble, Phys. Rev. B 9, 5236 (1974).

${ }^{19}$ P.R. Graves, C. Johnston, and J.J. Campaniello, Mater. Res. Bull. 23, 1651 (1988).

${ }^{20}$ L. Degiorgi, I. Blatter-Morke, and P. Wachter, Phys. Rev. B 35, 5421 (1987)

${ }^{21}$ B.N. Brockhouse and H. Watanabe, Phys. Lett. 1, 189 (1962).

${ }^{22}$ T.R. Hart, H. Temkin, and S.B. Adams, in Light Scattering in Solids, edited by M. Balkanski, R.C.C. Leite, and S.P.S. Porto (Flammarion Sciences, Paris, 1975), p. 254.

${ }^{23}$ R. Aragon, D.J. Buttrey, J.P. Shepherd, and J.M. Honig, Phys. Rev. B 31, 430 (1985); J.P. Shepherd et al., ibid. 31, 1107 (1985). 
${ }^{24}$ W.B. White and B.A.D. Angelis, Spectrochim. Acta, Part A 23, 985 (1967).

${ }^{25}$ R.D. Waldron, Phys. Rev. 99, 1727 (1955).

${ }^{26}$ W.C. Hamilton, Phys. Rev. 110, 1050 (1958).

${ }^{27}$ A. Kozlowski et al., J. Phys.: Condens. Matter 11, 2749 (1999).

${ }^{28}$ I.R. Beattie and T.R. Gilson, J. Chem. Soc. A 5, 980 (1970).

${ }^{29}$ L.R. Edwards and R.W. Lynch, J. Phys. Chem. Solids 31, 573 (1970).

${ }^{30}$ A.K. Sood, A.K. Arora, V. Umadevi, and G. Venkataraman,
Pramana, J. Phys. 16, 1 (1981).

${ }^{31}$ P.B. Allen, Solid State Commun. 14, 937 (1974).

${ }^{32}$ J.H. Park, L.H. Tjeng, J.W. Allen, P. Metcalf, and C.T. Chen, Phys. Rev. B 55, 12813 (1997).

${ }^{33}$ A. Chainani, T. Yokoya, T. Morimoto, T. Takahashi, and S. Todo, Phys. Rev. B 51, 17976 (1995).

${ }^{34}$ C.M. Varma, J. Zaanen, and K. Raghavachari, Science 254, 899 (1991). 